\title{
Comparison of Two Different Vinblastine Dosages for Treatment of Cutaneous Mast Cell Tumor in Dogs
}

\author{
Lucas Cavalli Kluthcovsky', Bruna Fernanda Firmo ${ }^{2}$, Pedro Carvalho Cassino ${ }^{2}$, Andrigo Barboza De Nardi ${ }^{2}$, \\ Jorge Luiz Costa Castro', Renata Luiza Halila' \& Jair Rodini Engracia Filho'
}

\begin{abstract}
Background: Mast cell tumors (MCT) are among the most common malignant cutaneous neoplasm in dogs with variable biologic behavior and remain a therapeutic challenge in high-grade cases. Surgery remains the primary treatment for canine MCT; however, chemotherapy and radiation therapy are commonly used to treat aggressive cases. The combination of vinblastine (VBL) at a dose of $2 \mathrm{mg} / \mathrm{m}^{2}$ and prednisone is the classically described protocol for MCT treatment. Studies have shown the safety of higher VBL doses for dogs with MCT, but there is a lack of information regarding dose intensity and outcome as a goal after chemotherapy. This study aimed to evaluate the impact of a higher dose of VBL on MCT treatment outcome.

Materials, Methods \& Results: This was an observational and comparative study conducted in two different Veterinary Teaching Hospitals. Client-owned dogs with histopathological diagnosis of grade II or III MCT were selected and underwent at least four chemotherapy sessions with VBL and prednisone. The experimental group (EG) consisted of 18 dogs that received a dose of $3 \mathrm{mg} / \mathrm{m}^{2}$ VBL treated in one institution. The control group (CG) included $31 \mathrm{dogs}$ that received a dose of $2 \mathrm{mg} / \mathrm{m}^{2}$ VBL treated at the other institution. All dogs treated in both groups had a clinical and complete blood count (CBC) evaluation performed previous the start of chemotherapy (T0) and before each weekly treatment (T1, T2, T3, and T4). After treatment, dogs in both groups were followed-up for the recurrence rate and overall survival time after diagnosis. There was no significant difference in clinical variables between EG and CG. During treatment, dogs of EG showed a significant reduction in erythrocyte, hemoglobin, and hematocrit values between T0 and T1, T2, T3, and T4 $(P<0.001)$. Comparatively, the CG showed significant reduction in hemoglobin $(P=0.02)$ and total leucocytes $(P=0.001)$ values in the same period. Despite these findings, these hematological parameters did not exceed the lower limit for the species in both groups. There was a higher-grade neutropenia one week after the first VBL application (T2) in both groups, with no statistical difference in neutrophil counts at $\mathrm{T} 2$ or during the whole treatment. There were discrete and self-limited episodes of anorexia, vomiting, and diarrhea in both groups. After chemotherapy, dogs in EG showed a significantly lower rate of recurrence than dogs in CG $(P=0.02)$. There was no significant difference in the overall survival time between groups. Discussion: The absence of significant differences in clinical variables (e.g. sex, age, histological grading, and tumor location) between EG and CG suggests that the groups may be similar regarding these parameters. All dogs included in this study had a recommendation for MCT post-operative chemotherapy treatment. VBL action is non-selective and anemia is not a commonly described adverse effect associated with its administration. Despite that, EG dogs exhibited a reduction in erythrocytes, hematocrit, and hemoglobin, and CG dogs in hemoglobin throughout T0 to T4. The highest number of neutropenia episodes occurred during T2, after the first VBL application in both groups with a trend of stabilization after $\mathrm{T} 2$, which is compatible with findings described in the literature. Any dog of EG or CG had to interrupt the treatment due to hematological or gastrointestinal toxicity or died during treatment. The role of VBL dose intensity in outcome is still debatable for dogs with MCT, once it is a multifactorial disease with variable presentation. In this study, there was no difference in overall survival time after diagnosis between groups, and EG dogs treated with a higher VBL dose showed significantly less tumor recurrence than CG.
\end{abstract}

Keywords: chemotherapy, dog, mast cell tumor, vinblastine.

DOI: $10.22456 / 1679-9216.101997$

${ }^{1}$ School of Life Sciences, Pontifical Catholic University of Parana (PUCPR), Curitiba, PR, Brazil. ${ }^{2}$ Department of Clinic and Veterinary Surgery, School of Agrarian and Veterinary Sciences, São Paulo State University (UNESP/FCAV), Jaboticabal, SP, Brazil. CORRESPONDENCE: L.C. Kluthcovksy [lucaskluth@gmail.com]. School of Life Sciences - PUCPR. Rua Imaculada Conceição n. 1155. CEP 80215-901 Curitiba, PR, Brazil. 


\section{INTRODUCTION}

Mast cell tumor (MCT) represents one of the most common malignant tumors in dogs. It consists in a proliferation of neoplastic mast cells with variable clinical presentation [6,17]. Histopathological examination is the main criteria for diagnosis [2]. Surgery remains the main choice for most cases of MCT. Chemotherapy and/or radiotherapy protocols are complementary treatments for high-grade tumors, incompletely excised MCTs, or disseminated/metastatic cases $[5,9,11]$. Tumor recurrence and metastasis are common problems, especially in high-grade tumors $[6,12,19]$.

Chemotherapy with VBL and prednisone has shown high efficacy in dogs with MCT, resulting in low recurrence rate and better overall survival time $[9,20,24]$. The most common protocol consists in VBL at $2 \mathrm{mg} / \mathrm{m}^{2}$, by intravenous route, for four weekly sessions, followed by four biweekly sessions. In addition, oral prednisone is combined at an initial dose of $2 \mathrm{mg} /$ $\mathrm{kg}$, with decreasing doses over time $[3,14]$.

Studies have demonstrated the safety of higher VBL doses [1,15,20,21]. Dogs treated with VBL at 3.5 $\mathrm{mg} / \mathrm{m}^{2}$ showed better overall response rate than those treated with $2 \mathrm{mg} / \mathrm{m}^{2}$, when the primary outcome was reduction in tumor size [20]. There are studies with escalating VBL doses for dogs, and the mean safe dosage ranged from $2.67 \mathrm{mg} / \mathrm{m}^{2}$ to $3.5 \mathrm{mg} / \mathrm{m}^{2}[1,21,23]$. However, there is a lack of evidence that higher VBL doses may provide long-term therapeutic gains. This study aimed to evaluate adverse effects during treatment, the recurrence rate and overall survival time after diagnosis in dogs with MCT treated with $3 \mathrm{mg} / \mathrm{m}^{2} \mathrm{VBL}$ (experimental group - EG), and compare to dogs from the control group (CG) treated with $2 \mathrm{mg} / \mathrm{m}^{2} \mathrm{VBL}$.

\section{MATERIALS AND METHODS}

\section{Study subjects}

This study was conducted in two different Veterinary Teaching Hospitals: The Veterinary Clinic School of the Pontifical Catholic University of Paraná (PUCPR), and at the Veterinary Hospital of the School of Agrarian and Veterinary Sciences, São Paulo State University (UNESP/FCAV), Jaboticabal, São Paulo, Brazil. This study involved an observational, comparative, and quantitative approach. The EG was composed of 18 dogs prospectively followed at the PUCPR Vet- erinary Clinic School. The CG was composed of 31 dogs that underwent treatment at the UNESP/FCAV Veterinary Hospital. The PUCPR Ethics Committee for Animal Use approved this project under protocol 01106/2016. All tutors of selected dogs signed a consent form.

\section{Patient Selection}

The EG group consisted of client-owned dogs diagnosed with MCT. The inclusion criteria were as follows: dogs with histopathological diagnosis of MCT, complete treatment with at least four weekly applications of $3 \mathrm{mg} / \mathrm{m}^{2}$ VBL (Faulblastina ${ }^{\circledR}$ Enj. Sol.) ${ }^{1}$ associated to prednisone $\left(\text { Meticorten }^{\circledR}\right)^{2}$, and dogs whose tutors agreed to participate in the study. Exclusion criteria were as follows: dogs diagnosed with MCT grade I/low grade; those with another type of tumor in addition to MCT; those with pre-existing chronic liver or kidney disease and/or cytopenias such as anemia, thrombocytopenia, and neutropenia. Thirty-two dogs with confirmed diagnosis of MCT were followed-up at the PUCPR Veterinary Clinic School. Of this total, five dogs did not undergo chemotherapy after surgery [due to death $(n=1)$ and refusal by the tutors $(n=4)$ ], four dogs did not complete the minimum treatment, and five had grade I/low grade MCT diagnosis, which resulted in 18 dogs selected for EG. The inclusion and exclusion criteria were the same for $\mathrm{CG}$, except that the dose of VBL used was $2 \mathrm{mg} / \mathrm{m}^{2}$. Thus, CG comprised of $31 \mathrm{dogs}$ treated at the UNESP/FCAV Veterinary Hospital during the study period.

\section{Diagnosis, staging, and surgical excision}

The following information were collected for the EG and CG as medical history: age in years, sex, weight in kilograms, reproductive status (intact or neutered), history of previous surgical excision, disease onset time, number of lesions, tumor location, and presence of cutaneous ulcerations. Dogs in both groups underwent physical examination. The initial staging workup comprised of a test for complete blood count (CBC), serum biochemical analysis (alanine aminotransferase, alkaline phosphatase, urea, creatinine, total protein, albumin, and glucose), nodule cytology, and abdominal ultrasonography. All dogs in the EG underwent surgical excision $(n=18)$. In CG, 29 dogs underwent surgery for tumor removal, and two dogs had MCT classified as unresectable. After surgery, dogs were followed-up regularly during the postoperative period 
to detect possible postoperative complications such as wound dehiscence, necrosis, ulceration, and local or distant recurrence. After surgery or biopsy, all tumors were evaluated by histopathological examination and classified using previously described grading systems $[7,13]$. In cases where the patient had multiple tumors with different grades, the highest grade was recorded.

\section{Treatment and toxicity assessment}

In both groups, chemotherapy consisted of at least four sessions each week, involving the intravenous administration of VBL. EG dogs received a VBL dose of $3 \mathrm{mg} / \mathrm{m}^{2}$, while dogs in the $\mathrm{CG}$ received $2 \mathrm{mg} / \mathrm{m}^{2}$ VBL per session. Dogs in both groups received oral prednisone at an initial dose of $2 \mathrm{mg} / \mathrm{kg}$ per day. There was a gradual weekly corticosteroid dose reduction to $0.5 \mathrm{mg} / \mathrm{kg}$ per day, maintained until the end of treatment. All dogs treated in both groups had a clinical and hematological evaluation by CBC performed before treatment (Time 0, T0). After the start of chemotherapy, all dogs had a CBC evaluation before each treatment, for a period of four weeks (T1, T2, T3, and T4).

The occurrence of gastrointestinal adverse effects such as anorexia, vomiting, and diarrhea was recorded at each visit. Gastrointestinal toxicity was evaluated and graded from 0 to 5 , as previously described [22]. CBC data involving blood counts, leukogram, and platelet values revealed possible hematological toxicity. In both groups, treatment was postponed for at least seven days if there was pretreatment grade 2 or higher neutropenia or gastrointestinal toxicity $\geq$ grade 2 dogs [22].

\section{Recurrence rate and survival time}

Dogs in the EG and CG were followed-up until the end of the study. The objective was to record local or distant recurrence, new localized or disseminated skin lesions, survival time, and occurrence of deaths. The team contacted the dog tutors by telephone for updates on patients that could not be followed-up; four dogs from the CG could not be followed-up until the end of the study. Information about recurrence after treatment and survival time was collected for 25 and 27 of the $31 \mathrm{CG}$ dogs, respectively. For EG dogs, data regarding recurrence after treatment and survival time was collected for all 18 cases. A comparison between the overall survival curves of the EG and CG dogs was performed, from the time between MCT diagnosis until the last follow-up or death.

\section{Statistical analysis}

Absolute and relative frequencies and descriptive statistical measures were used for analyses of clinical and treatment variables. Descriptive statistics are reported as mean (standard deviation). Fisher's exact test and Pearson's Chi-square test were used to evaluate the association between categorical variables, and evaluation of adjusted residuals for variables with three or more categories. The Student's t-test and Mann-Whitney test compared two independent groups. Comparisons over the treatment period (T0 to T4) were performed using the repeated measures analysis of variance (ANOVA) test, with Bonferroni correction, repeated measures Student's $t$-test, and Friedman and Wilcoxon tests. Data gaussianity was verified using histograms and the Shapiro-Wilk test.

Multivariate ANOVA for repeated measures was used to compare hematological parameters during treatment ( $\mathrm{T} 0$ to $\mathrm{T} 4$ ) between groups (EG and $\mathrm{CG})$. This test was applied only to variables that were significantly different in the bivariate analysis for both groups (hemoglobin and eosinophils).

Comparison of the recurrence rate after treatment between EG and CG was calculated using the Pearson Chi-square and then adjusted by logistic regression. Overall survival curves of follow-up after MCT diagnosis were compared between EG and CG dogs. Dogs with the last registered follow-up (censorship) and deaths (case) were considered. Survival functions were calculated using the Kaplan-Meier survival analysis with log-rank tests. The software used for statistical analysis was the Statistical Package for Social Science (SPSS), version 15.0 and the level of significance was set at $P<0.05$ for all tests.

\section{RESULTS}

Among the 18 dogs in the EG, 10 (55.6\%) were females and eight (44.4\%) were males. Breeds included mixed ( $n=7)$, Labrador retriever $(n=4)$, and one each of the following: Boxer, Brazilian Terrier, Pinscher, Pit Bull, Poodle, Rottweiler and Shar-Pei. Of the 31 CG dogs, 20 $(64.5 \%)$ were females and 11 (35.5\%) were males. Breeds included mixed ( $n=9)$, Boxer ( $n=4)$, Dachshund $(n=4)$, Labrador Retriever $(n=3)$, Pit Bull $(n=3)$, Pinscher $(n=$ $2)$, Pug $(n=2)$ and one each of the following: Bernese Mountain Dog, Brazilian Terrier, Doberman, and Golden Retriever. The distribution of EG and CG according to clinical variables is presented in Table 1 . The variables were not significantly different between groups. 
Table 1. Distribution of Experimental and Control Groups according to clinical variables.

\begin{tabular}{|c|c|c|c|c|c|}
\hline \multirow[t]{2}{*}{ Variable } & \multicolumn{2}{|c|}{$\begin{array}{l}\text { Experimental Group } \\
\qquad(\mathrm{n}=18)\end{array}$} & \multicolumn{2}{|c|}{$\begin{array}{l}\text { Control Group } \\
\quad(\mathrm{n}=31)\end{array}$} & \multirow[t]{2}{*}{$P$} \\
\hline & Mean (SD) & $\mathrm{n}(\%)$ & Mean (SD) & $\mathrm{n}(\%)$ & \\
\hline Age (years) & $8.8(2.1)$ & & $8.4(3.0)$ & & $0.92 *$ \\
\hline Sex & & & & & $0.56^{* *}$ \\
\hline Female & & $10(55.6)$ & & $20(64.5)$ & \\
\hline Male & & $8(44.4)$ & & $11(35.5)$ & \\
\hline Reproductive status & & & & & $0.76^{* * *}$ \\
\hline Intact & & $7(38.9)$ & & $10(32.3)$ & \\
\hline Neutered & & $11(61.1)$ & & $21(67.7)$ & \\
\hline Tumor recurrence prior vinblastine chemotherapy & & & & & $0.14 * *$ \\
\hline Yes & & $6(33.3)$ & & $4(12.9)$ & \\
\hline No & & $12(66.7)$ & & $27(87.1)$ & \\
\hline Onset time (months) & $3.8(3.9)$ & & $5.6(8.6)$ & & $0.50 *$ \\
\hline Number of lesions & & & & & $0.53 * *$ \\
\hline 1 & & $10(55.6)$ & & $20(64.5)$ & \\
\hline$\geq 2$ & & $8(44.4)$ & & $11(35.5)$ & \\
\hline Tumor location & & & & & $0.61 * *$ \\
\hline Head/neck & & $2(11.1)$ & & $8(25.8)$ & \\
\hline Trunk & & $8(44.4)$ & & $12(38.7)$ & \\
\hline Extremity & & $6(33.3)$ & & $7(22.6)$ & \\
\hline Inguinal/perineal & & $2(11.1)$ & & $4(12.9)$ & \\
\hline Skin ulceration & & & & & $0.18 * *$ \\
\hline Present & & $8(44.4)$ & & $8(25.8)$ & \\
\hline Absent & & $10(55.6)$ & & $23(74.2)$ & \\
\hline Sign of abdominal metastasis & & & & & $0.07 * * *$ \\
\hline Present & & $0(0.0)$ & & $6(19.4)$ & \\
\hline Absent & & $18(100.0)$ & & $25(80.6)$ & \\
\hline Histological grade ${ }^{\dagger}$ & & & & & $0.66^{* * *}$ \\
\hline II & & $15(83.3)$ & & $28(90.3)$ & \\
\hline III & & $3(16.7)$ & & $3(9.7)$ & \\
\hline Histological grade ${ }^{\ddagger}$ & & & & & $0.55 * *$ \\
\hline Low-grade & & $12(66.7)$ & & $18(58.1)$ & \\
\hline High-grade & & $6(33.3)$ & & 13(41.9) & \\
\hline
\end{tabular}

$†$ Reference 13; $†$ Reference 7 ; *Mann-Whitney test, $P<0.05$; **Chi-square test, $P<0.05$; ***Fisher’s exact test, $P<0.05$.

Table 2 shows the distribution of CBC parameters of EG at the beginning of treatment and during the subsequent four sessions. There was a significant reduction in erythrocyte, hemoglobin, hematocrit, and eosinophil counts between T0 and T1, T2, T3, and T4 $(P<0.001)$. However, there was no significant difference between $\mathrm{T} 0$ and T1 for erythrocytes, hemoglobin and eosinophils. In addition, there was no significant difference between $\mathrm{T} 0$ and $\mathrm{T} 3$ for erythrocytes and hemoglobin. There was a significant reduction in erythrocytes, hemoglobin, hematocrit and eosinophils between $\mathrm{T} 0$ and $\mathrm{T} 2$, and $\mathrm{T} 0$ and $\mathrm{T} 4$. The monocyte count increased significantly between $\mathrm{T} 0$ and $\mathrm{T} 2$ and between $\mathrm{T} 0$ and T3. Despite the variations in the aforementioned $\mathrm{CBC}$ parameters, the means were in the reference range for the species.

Table 3 shows the distribution of CBC parameters of $\mathrm{CG}$ dogs during treatment. There was a significant difference in hemoglobin levels throughout the sessions, but no difference in specific comparison between $\mathrm{T} 0$ and $\mathrm{T} 1, \mathrm{~T} 0$ and $\mathrm{T} 2, \mathrm{~T} 0$ and $\mathrm{T} 3$, and between $\mathrm{T} 0$ and $\mathrm{T} 4$. There was a significant reduction in total leukocyte and lymphocyte counts between $\mathrm{T} 0$ and $\mathrm{T} 2$ and between $\mathrm{T} 0$ and T3. Eosinophil count showed significant reduction between T0 and T2, T3, and T4. Despite these variations, all the means and medians were within the reference range for dogs. There was no significant difference in neutrophils counts during the treatment for both groups.

When the EG and CG were compared using multivariate ANOVA for repeated measures, there was a significant reduction in hemoglobin $(P<0.001)$ and eosinophils $(P<0.001)$ in both groups during the four-week chemotherapy period. However, there was no significant difference in the values of these parameters between EG and CG when the VBL dose was considered as a dependent variable ( $P=0.38$ for hemoglobin and $P=0.86$ for eosinophils). 
Table 2. Distribution of hematological parameters of $18 \mathrm{dogs}$ treated with $3 \mathrm{mg} / \mathrm{m}^{2}$ vinblastine (EG) before the start of chemotherapy treatment (T0) and during the subsequent four sessions (T1 to T4).

\begin{tabular}{|c|c|c|c|c|c|c|c|c|}
\hline \multirow{2}{*}{ Parameter } & $\begin{array}{l}\text { Start } \\
(\mathrm{T} 0)\end{array}$ & $\begin{array}{c}1^{\text {st }} \text { week } \\
\text { (T1) }\end{array}$ & $\begin{array}{c}2^{\text {nd }} \text { week } \\
\text { (T2) }\end{array}$ & $\begin{array}{c}3^{\text {rd }} \text { week } \\
\text { (T3) }\end{array}$ & $\begin{array}{c}4^{\text {th }} \text { week } \\
\text { (T4) }\end{array}$ & \multirow{2}{*}{$P$} & \multirow{2}{*}{ T0 x Tn } & \multirow{2}{*}{$P$} \\
\hline & $\begin{array}{l}\text { Mean } \\
(\mathrm{SD})^{\dagger}\end{array}$ & $\begin{array}{l}\text { Mean } \\
(\mathrm{SD})^{\dagger}\end{array}$ & $\begin{array}{l}\text { Mean } \\
(\mathrm{SD})^{\dagger}\end{array}$ & $\begin{array}{l}\text { Mean } \\
(\mathrm{SD})^{\dagger}\end{array}$ & $\begin{array}{l}\text { Mean } \\
(\mathrm{SD})^{\dagger}\end{array}$ & & & \\
\hline \multirow[t]{4}{*}{ Erythrocytes $\left(10^{6} / \mu \mathrm{L}\right)$} & $\begin{array}{c}6.8 \\
(1.0)\end{array}$ & $\begin{array}{c}6.4 \\
(1.2)\end{array}$ & $\begin{array}{c}6.0 \\
(1.2)\end{array}$ & $\begin{array}{c}6.0 \\
(1.2)\end{array}$ & $\begin{array}{c}5.5 \\
(1.3)\end{array}$ & $<0.001 *$ & $\mathrm{~T} 0>\mathrm{T} 1$ & $0.78 * *$ \\
\hline & & & & & & & $\mathrm{T} 0>\mathrm{T} 2$ & $0.04 * *$ \\
\hline & & & & & & & $\mathrm{T} 0>\mathrm{T} 3$ & $0.07 * *$ \\
\hline & & & & & & & $\mathrm{T} 0>\mathrm{T} 4$ & $0.001 * *$ \\
\hline \multirow[t]{4}{*}{ Hemoglobin (g/dL) } & $\begin{array}{l}16.0 \\
(2.2)\end{array}$ & $\begin{array}{l}15.2 \\
(2.4)\end{array}$ & $\begin{array}{l}14.1 \\
(2.6)\end{array}$ & $\begin{array}{l}14.3 \\
(2.8)\end{array}$ & $\begin{array}{l}13.4 \\
(3.2)\end{array}$ & $<0.001 *$ & $\mathrm{~T} 0>\mathrm{T} 1$ & $0.89 * *$ \\
\hline & & & & & & & $\mathrm{T} 0>\mathrm{T} 2$ & $0.03 * *$ \\
\hline & & & & & & & $\mathrm{T} 0>\mathrm{T} 3$ & $0.06 * *$ \\
\hline & & & & & & & $\mathrm{T} 0>\mathrm{T} 4$ & $0.001 * *$ \\
\hline \multirow[t]{4}{*}{ Hematocrit (\%) } & $\begin{array}{l}46.7 \\
(5.6)\end{array}$ & $\begin{array}{l}44.2 \\
(7.0)\end{array}$ & $\begin{array}{l}41.4 \\
(6.7)\end{array}$ & $\begin{array}{l}40.8 \\
(6.7)\end{array}$ & $\begin{array}{l}38.8 \\
(8.5)\end{array}$ & $<0.001 *$ & $\mathrm{~T} 0>\mathrm{T} 1$ & $0.04 * *$ \\
\hline & & & & & & & $\mathrm{T} 0>\mathrm{T} 2$ & $0.01 * *$ \\
\hline & & & & & & & $\mathrm{T} 0>\mathrm{T} 3$ & $<0.01 * *$ \\
\hline & & & & & & & $\mathrm{T} 0>\mathrm{T} 4$ & $0.001 * *$ \\
\hline Platelets $\left(10^{3} / \mu \mathrm{L}\right)$ & $\begin{array}{c}302.7 \\
(118.1)\end{array}$ & $\begin{array}{c}330.7 \\
(136.2) \\
\end{array}$ & $\begin{array}{l}304.9 \\
(98.0) \\
\end{array}$ & $\begin{array}{l}313.7 \\
(97.6) \\
\end{array}$ & $\begin{array}{l}300.7 \\
(75.2) \\
\end{array}$ & $0.81 *$ & - & - \\
\hline Leukocytes $\left(10^{3} / \mu \mathrm{L}\right)$ & $\begin{array}{r}12.3 \\
(9.0) \\
\end{array}$ & $\begin{array}{l}12.2 \\
(6.0) \\
\end{array}$ & $\begin{array}{c}12.9 \\
(14.9) \\
\end{array}$ & $\begin{array}{c}15.7 \\
(19.0) \\
\end{array}$ & $\begin{array}{c}16.4 \\
(18.9) \\
\end{array}$ & $0.05 * * *$ & - & - \\
\hline \multirow[t]{4}{*}{ Eosinophils $(/ \mu \mathrm{L})$} & $\begin{array}{c}438.9 \\
(359.6)\end{array}$ & $\begin{array}{c}504.4 \\
(441.0)\end{array}$ & $\begin{array}{c}43.1 \\
(55.2)\end{array}$ & $\begin{array}{c}157.9 \\
(218.9)\end{array}$ & $\begin{array}{c}183.3 \\
(259.3)\end{array}$ & $<0.001 * * *$ & $\mathrm{~T} 0>\mathrm{T} 1$ & $0.40 * *$ \\
\hline & & & & & & & $\mathrm{T} 0>\mathrm{T} 2$ & $0.001 * * * *$ \\
\hline & & & & & & & $\mathrm{T} 0>\mathrm{T} 3$ & $0.02 * * * *$ \\
\hline & & & & & & & $\mathrm{T} 0>\mathrm{T} 4$ & $0.01 * * * *$ \\
\hline 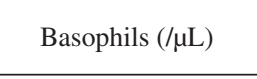 & $\begin{array}{c}6.3 \\
(26.9) \\
\end{array}$ & $\begin{array}{c}53.5 \\
(159.9) \\
\end{array}$ & $\begin{array}{c}5.4 \\
(22.9) \\
\end{array}$ & $\begin{array}{c}0.0 \\
(0.0) \\
\end{array}$ & $\begin{array}{c}0.0 \\
(0.0) \\
\end{array}$ & $0.34 * * *$ & - & - \\
\hline Lymphocytes $(/ \mu \mathrm{L})$ & $\begin{array}{c}1700.2 \\
(1047.9) \\
\end{array}$ & $\begin{array}{r}1483.6 \\
(927.9) \\
\end{array}$ & $\begin{array}{c}1539.1 \\
(1119.7) \\
\end{array}$ & $\begin{array}{c}1962.4 \\
(1397.2) \\
\end{array}$ & $\begin{array}{l}1608.9 \\
(991.8) \\
\end{array}$ & $0.31 * * *$ & - & - \\
\hline \multirow[t]{4}{*}{ Monocytes $(/ \mu \mathrm{L})$} & $\begin{array}{c}699.5 \\
(792.4)\end{array}$ & $\begin{array}{c}646.3 \\
(467.3)\end{array}$ & $\begin{array}{c}1115.4 \\
(1176.0)\end{array}$ & $\begin{array}{c}1222.5 \\
(1299.6)\end{array}$ & $\begin{array}{c}2006.0 \\
(4307.6)\end{array}$ & $0.03 * * *$ & $\mathrm{~T} 0<\mathrm{T} 1$ & $0.79 * * * *$ \\
\hline & & & & & & & $\mathrm{T} 0<\mathrm{T} 2$ & $0.02 * * * *$ \\
\hline & & & & & & & $\mathrm{T} 0<\mathrm{T} 3$ & $0.01 * * * *$ \\
\hline & & & & & & & $\mathrm{T} 0<\mathrm{T} 4$ & $0.36^{* * * * *}$ \\
\hline Neutrophils $(/ \mu \mathrm{L})$ & $\begin{array}{l}10005.0 \\
(7995.2)\end{array}$ & $\begin{array}{c}9575.4 \\
(5890.2)\end{array}$ & $\begin{array}{c}10203.4 \\
(13274.7)\end{array}$ & $\begin{array}{c}12325.6 \\
(18017.4)\end{array}$ & $\begin{array}{c}12629.5 \\
(16391.3)\end{array}$ & $0.08 * * *$ & - & - \\
\hline
\end{tabular}

*Repeated measures ANOVA with Bonferroni correction; **Dependent $t$-test; ***Friedman test; ****Wilcoxon test; $\uparrow \mathrm{SD}=$ standard deviation.

In both groups, there was higher-grade neutropenia one week after the first VBL application (T2). There was no significant difference in neutrophil counts between the groups at T2 $(P=0.40$; Table 4$)$. At T2, the EG group showed five dogs $(27.8 \%)$ with some grade of neutropenia: one dog $(5.6 \%)$ with neutropenia grade 1, 2 and 4, and two dogs with neutropenia grade $3(11.1 \%)$. The number of neutropenic dogs from EG was also five at T3, with different distribution in grades: four dogs with neutropenia grade 1 , and one dog with neutropenia grade 2 . In comparison, the CG showed reduction from five neutropenic dogs in $\mathrm{T} 2$ (three grade 1 , one grade 2 , and one grade 3 neutropenia) to four in
T3 (all cases classified as grade 1 neutropenia). Prior to the fourth session (T4), one (5.6\%) EG dog presented grade 1 neutropenia and two $(6.4 \%)$ CG dogs had grade 2 neutropenia. Platelet values remained stable in most dogs in both the groups. At T1, only one EG $\operatorname{dog}(5.6 \%)$ and two CG dogs (3.2\%) presented grade 1 thrombocytopenia.

In the EG, eight dogs (44.4\%) presented gastrointestinal adverse effects classified as grade 1 anorexia $(n=1 ; 5.5 \%)$, grade 1 vomiting $(n=4 ; 22.0 \%)$, grade 1 diarrhea $(n=1 ; 5.5 \%)$, and one $\operatorname{dog}(5.5 \%)$ presented grade 2 diarrhea after the first session. In the CG, nine dogs $(29.0 \%)$ presented gastrointestinal toxicity classi- 
Table 3. Distribution of hematological parameters of 31 dogs treated with $2 \mathrm{mg} / \mathrm{m}^{2}$ vinblastine (CG) before the start of chemotherapy treatment (T0) and during the subsequent four sessions (T1 to T4).

\begin{tabular}{|c|c|c|c|c|c|c|c|c|}
\hline Parameter & $\begin{array}{l}\begin{array}{l}\text { Start } \\
(\mathrm{T} 0)\end{array} \\
\text { Mean } \\
(\mathrm{SD})^{\dagger}\end{array}$ & $\begin{array}{c}1^{\text {st }} \text { week } \\
(\mathrm{T} 1) \\
\text { Mean } \\
(\mathrm{SD})^{\dagger}\end{array}$ & $\begin{array}{c}2^{\text {nd }} \text { week } \\
(\mathrm{T} 2)\end{array}$ & $\begin{array}{c}3^{\text {rd }} \text { week } \\
\text { (T3) } \\
\text { Mean } \\
(\mathrm{SD})^{\dagger}\end{array}$ & $\begin{array}{c}4^{\text {th }} \text { week } \\
(\mathrm{T} 4) \\
\text { Mean } \\
(\mathrm{SD})^{\dagger}\end{array}$ & $P$ & T0 x Tn & $P$ \\
\hline Erythrocytes $\left(10^{6} / \mu \mathrm{L}\right)$ & $\begin{array}{c}6.4 \\
(1.2) \\
\end{array}$ & $\begin{array}{c}6.4 \\
(1.3) \\
\end{array}$ & $\begin{array}{c}6.2 \\
(1.0) \\
\end{array}$ & $\begin{array}{c}6.3 \\
(0.9) \\
\end{array}$ & $\begin{array}{c}6.1 \\
(0.8) \\
\end{array}$ & $0.24 *$ & - & - \\
\hline \multirow[t]{4}{*}{ Hemoglobin $(\mathrm{g} / \mathrm{dL})$} & $\begin{array}{l}15.6 \\
(3.1)\end{array}$ & $\begin{array}{l}15.7 \\
(3.0)\end{array}$ & $\begin{array}{l}15.0 \\
(2.6)\end{array}$ & $\begin{array}{l}14.9 \\
(2.2)\end{array}$ & $\begin{array}{l}14.9 \\
(2.1)\end{array}$ & $0.02 *$ & $\mathrm{~T} 0<\mathrm{T} 1$ & $1.00 * * *$ \\
\hline & & & & & & & $\mathrm{T} 0>\mathrm{T} 2$ & $0.46^{* * *}$ \\
\hline & & & & & & & $\mathrm{T} 0>\mathrm{T} 3$ & $0.60 * * *$ \\
\hline & & & & & & & $\mathrm{T} 0>\mathrm{T} 4$ & $1.00^{* * *}$ \\
\hline Hematocrit (\%) & $\begin{array}{l}43.8 \\
(9.0) \\
\end{array}$ & $\begin{array}{l}44.1 \\
(9.2) \\
\end{array}$ & $\begin{array}{l}42.1 \\
(7.4) \\
\end{array}$ & $\begin{array}{l}42.7 \\
(6.8) \\
\end{array}$ & $\begin{array}{l}41.6 \\
(5.4) \\
\end{array}$ & $0.06^{*}$ & - & - \\
\hline Platelets $\left(10^{3} / \mu \mathrm{L}\right)$ & $\begin{array}{c}390.2 \\
(246.7) \\
\end{array}$ & $\begin{array}{c}407.7 \\
(166.6) \\
\end{array}$ & $\begin{array}{c}448.3 \\
(226.5) \\
\end{array}$ & $\begin{array}{c}404.4 \\
(187.7) \\
\end{array}$ & $\begin{array}{c}508.3 \\
(527.5) \\
\end{array}$ & $0.56^{* *}$ & - & - \\
\hline \multirow[t]{4}{*}{ Leukocytes $\left(10^{3} / \mu \mathrm{L}\right)$} & $\begin{array}{l}10.4 \\
(4.4)\end{array}$ & $\begin{array}{c}9.1 \\
(3.7)\end{array}$ & $\begin{array}{c}6.8 \\
(2.9)\end{array}$ & $\begin{array}{c}8.4 \\
(4.6)\end{array}$ & $\begin{array}{c}8.5 \\
(4.3)\end{array}$ & $0.001 * *$ & $\mathrm{~T} 0>\mathrm{T} 1$ & $0.18 * * * *$ \\
\hline & & & & & & & $\mathrm{T} 0>\mathrm{T} 2$ & $<0.001 * * * *$ \\
\hline & & & & & & & $\mathrm{T} 0>\mathrm{T} 3$ & $0.01 * * * *$ \\
\hline & & & & & & & $\mathrm{T} 0>\mathrm{T} 4$ & $0.06^{* * * * *}$ \\
\hline \multirow[t]{4}{*}{ Eosinophils $(/ \mu \mathrm{L})$} & $\begin{array}{c}584.3 \\
(676.4)\end{array}$ & $\begin{array}{c}435.9 \\
(531.0)\end{array}$ & $\begin{array}{c}84,7 \\
(255.1)\end{array}$ & $\begin{array}{c}110.4 \\
(123.1)\end{array}$ & $\begin{array}{c}178.5 \\
(265.2)\end{array}$ & $<0.001 * *$ & $\mathrm{~T} 0>\mathrm{T} 1$ & $0.18^{* * * * *}$ \\
\hline & & & & & & & $\mathrm{T} 0>\mathrm{T} 2$ & $<0.001 * * * *$ \\
\hline & & & & & & & $\mathrm{T} 0>\mathrm{T} 3$ & $<0.001 * * * *$ \\
\hline & & & & & & & $\mathrm{T} 0>\mathrm{T} 4$ & $<0.001 * * * *$ \\
\hline Basophils $(/ \mu \mathrm{L})$ & $\begin{array}{c}0.0 \\
(0.0) \\
\end{array}$ & $\begin{array}{c}14.2 \\
(67.1) \\
\end{array}$ & $\begin{array}{c}15.9 \\
(63.4) \\
\end{array}$ & $\begin{array}{c}16.5 \\
(92.0) \\
\end{array}$ & $\begin{array}{c}6.8 \\
(27.2) \\
\end{array}$ & $0.52^{* *}$ & - & - \\
\hline \multirow[t]{4}{*}{ Lymphocytes $(/ \mu \mathrm{L})$} & $\begin{array}{l}1390.5 \\
(672.8)\end{array}$ & $\begin{array}{c}1599.7 \\
(2066.3)\end{array}$ & $\begin{array}{c}1542.4 \\
(2277.2)\end{array}$ & $\begin{array}{l}1066.7 \\
(746.6)\end{array}$ & $\begin{array}{c}1359.2 \\
(1157.2)\end{array}$ & $0.02 * *$ & $\mathrm{~T} 0>\mathrm{T} 1$ & $0.30 * * * *$ \\
\hline & & & & & & & $\mathrm{T} 0>\mathrm{T} 2$ & $0.02 * * * *$ \\
\hline & & & & & & & $\mathrm{T} 0>\mathrm{T} 3$ & $0.01 * * * *$ \\
\hline & & & & & & & $\mathrm{T} 0>\mathrm{T} 4$ & $0.27 * * * *$ \\
\hline Monocytes $(/ \mu \mathrm{L})$ & $\begin{array}{c}292.0 \\
(224.4) \\
\end{array}$ & $\begin{array}{c}252.7 \\
(225.9) \\
\end{array}$ & $\begin{array}{c}332.8 \\
(380.1) \\
\end{array}$ & $\begin{array}{c}337.0 \\
(324.4) \\
\end{array}$ & $\begin{array}{c}299.9 \\
(248.7) \\
\end{array}$ & $0.97 * *$ & - & - \\
\hline Neutrophils $(/ \mu \mathrm{L})$ & $\begin{array}{c}8021.6 \\
(4229.7) \\
\end{array}$ & $\begin{array}{c}7159.2 \\
(3604.4) \\
\end{array}$ & $\begin{array}{c}5403.6 \\
(2712.0) \\
\end{array}$ & $\begin{array}{c}7031.7 \\
(4290.0) \\
\end{array}$ & $\begin{array}{c}6873.4 \\
(4225.2) \\
\end{array}$ & $0.15^{* *}$ & - & - \\
\hline
\end{tabular}

*Repeated measures ANOVA with Bonferroni correction; **Friedman test; ***Dependent $t$-test; ****Wilcoxon test; $† \mathrm{SD}=$ standard deviation.

Table 4. Distribution of experimental and control dogs, seven days after the first vinblastine application (T2), according to grades of neutropenia.

\begin{tabular}{ccc}
\hline & \multicolumn{2}{c|}{ Treatment Protocol (T2) } \\
\cline { 2 - 3 } Grade of neutropenia & $\begin{array}{c}\text { Experimental Group }(\mathrm{n}=18)^{*} \\
\mathrm{n}(\%)\end{array}$ & $\begin{array}{c}\text { Control Group }(\mathrm{n}=31)^{*} \\
\mathrm{n}(\%)\end{array}$ \\
\hline No neutropenia & $13(72.2)$ & $26(83.9)$ \\
$1(1500 / \mu \mathrm{L}-\mathrm{LLN})$ & $1(5.6)$ & $3(9.7)$ \\
$2(1000-1499 / \mu \mathrm{L})$ & $1(5.6)$ & $1(3.2)$ \\
$3(500-999 / \mu \mathrm{L})$ & $2(11.1)$ & $1(3.2)$ \\
$4(<500 / \mu \mathrm{L})$ & $1(5.6)$ & $0(0.0)$ \\
\hline
\end{tabular}

"Non-significant difference following the Pearson Chi-square test $(P=0.40) ; \mathrm{LLN}=$ lower limit of normal (3000 neutrophils $/ \mu \mathrm{L})$ 
fied as grade 1 anorexia $(n=2 ; 6.5 \%)$, grade 1 vomiting $(n=2 ; 6.5 \%)$, grade 1 diarrhea $(n=3 ; 9.7 \%)$, and grade 2 diarrhea $(n=2 ; 6.5 \%)$. These adverse effects were mild and self-limited in both groups and there was no interruption in treatment in any groups due to gastrointestinal toxicity.

After chemotherapy, tumor recurrence was observed in four of the $18 \mathrm{dogs}$ in the EG (22.2\%) and in 13 of the 25 dogs in the CG (52.0\%), with significant difference $(P=0.02)$ between groups.

Figure 1 shows the overall survival curves for the two groups. There was no significant difference in the overall survival between the groups $(P=0.11)$. A cross-sectional analysis of the overall survival of the dogs showed that over the period of 365 days (one year) after the histopathological diagnosis, the overall survival for the EG was $66.2 \%$ and that for the CG was $80.9 \%$; there was no significant difference between the groups $(P=0.05)$.

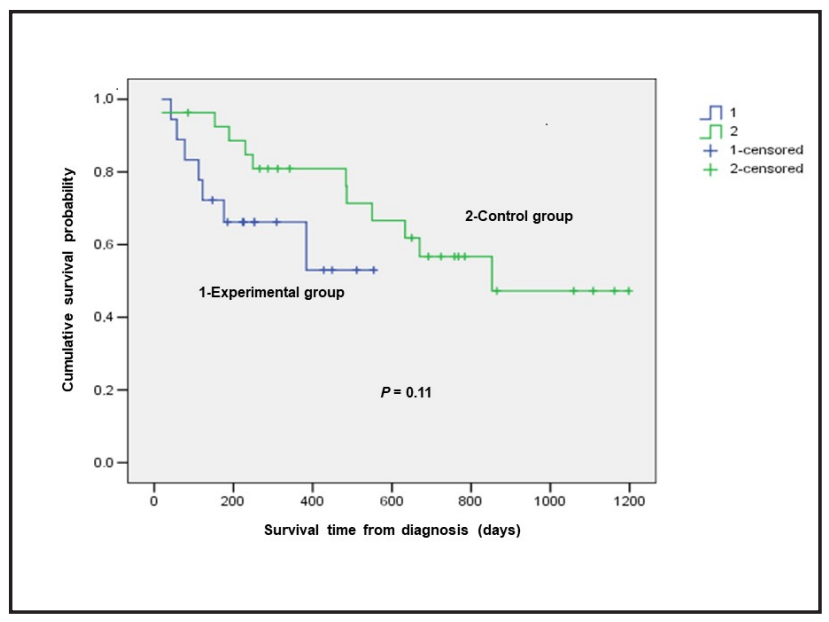

Figure 1. Kaplan-Meier survival curve for dogs of Experimental Group and Control Group follow-up after diagnosis.

\section{DISCUSSION}

This study evaluated the treatment of MCT in dogs in two groups: one group treated with a higher VBL dose $\left(3 \mathrm{mg} / \mathrm{m}^{2}\right)$ and the other group treated with the classic dose $\left(2 \mathrm{mg} / \mathrm{m}^{2}\right)$. There were no significant differences following comparison of the clinical variables between groups. These finding suggests that the groups may be similar regarding these variables. Initial exclusion of dogs with grade I MCT in both groups was based on previously published recommendations for chemotherapy treatment, once dogs with grade I/ low-grade MCT usually have a more favorable outcome when compared to those high-grade or grades
II/III MCT $[2,17,19]$. The mean ages for middle-aged dogs and elderly dogs for both groups were similar. These ages correspond to the age of MCT occurrence in dogs $[10,15,16]$. The frequency of high-grade tumors increases significantly mainly in elderly dogs with relapse. This possibly occurs due to the increased number of additional mutations over time [10].

The hematological parameters of the EG and CG were analyzed separately just before the start of treatment until the fourth session with VBL and prednisone. Currently, the use of VBL and prednisone in chemotherapy is recommended for the management of dogs with MCT, especially in cases involving highgrade tumors, unresectable tumors, and compromised surgical margins after excision [17]. VBL is a vinca alkaloid that acts during the mitosis stage of the cell cycle. It binds to protein microtubules in the mitotic spindle, eventually preventing cell replication and reducing mitosis. This action is non-selective, and may cause cytotoxicity in healthy and neoplastic cells [25]. Therefore, dogs undergoing VBL treatment should be evaluated regularly using the $\mathrm{CBC}$ to identify possible cytopenias, mainly anemia, neutropenia, and thrombocytopenia. Other adverse effects that may be observed during treatment are gastrointestinal adverse effects, which include anorexia, vomiting, and diarrhea $[1,12]$.

The occurrence of anemia is a constant concern in patients undergoing chemotherapy treatment in veterinary oncology [8]. In this study, dogs in both groups exhibited reduction in at least one parameter associated with the erythrogram. However, the hemoglobin, erythrocyte, and hematocrit values did not exceed the lower reference limits for the species. There was no identification of severe anemia during the treatment in both groups. Significant reduction in hematocrit or other erythrogram parameters was not previously described as a common adverse effect during VBL treatment $[1,15,20]$. In the $C G$, there was a significant reduction in the hemoglobin values over the four sessions. However, these values were not significantly correlated with the initial value observed at T0.

It is important to emphasize that groups were compared using multivariate analysis of variance using variables that were significant following the bivariate analysis (hemoglobin and eosinophils) between $\mathrm{T} 0$ and T4. There was significant reduction in hemoglobin and eosinophil in both groups during the treatment period. However, there was no significant difference in the 
values of these hematological parameters between the EG and CG when the VBL dose was used as the dependent variable. These results may suggest that both the usual dose and the highest dose of VBL result in similar hematological toxicity between groups.

The cytotoxic effect of VBL is more evident in cells with a high replication rate, such as neutrophils [20]. In the present study, the highest number of neutropenia episodes occurred during T2, after the first VBL application in both groups. The neutrophil values tended to stabilize after T2 and there was less occurrence of neutropenia after the second chemotherapy session. This finding was observed in previous studies, which also showed a trend towards stabilization of neutrophil values after treatment started $[3,20]$. Temporary interruption of treatment is recommended whenever CBC shows significant neutropenia, this will allow normalization of neutrophil values and avoid possible side effects due to immunosuppression [2,20,22].

In a previous study, administration of VBL at a dose of $3.5 \mathrm{mg} / \mathrm{m}^{2}$ caused grade 4 neutropenia in $46 \%$ of 26 treated dogs. The main outcome of the study was the evaluation of tumor size using VBL as a single agent for treatment. The response rate observed in the group of dogs receiving a dose of $2 \mathrm{mg} / \mathrm{m}^{2}$ was $11.8 \%$, while the $3.5 \mathrm{mg} / \mathrm{m}^{2}$ group had a response rate of $27 \%$ [14]. In the present study, although some dogs had macroscopic disease during treatment, the response rate relative to the reduction in tumor size was not the primary outcome.

Although adverse effects are generally mild during VBL chemotherapy, there is a description of a dog that exhibited grade 4 neutropenia, subsequently resulting in death due to sepsis during VBL treatment [3]. In the present study, no dog in the EG and $\mathrm{CG}$ died during treatment. Also, we detected grade 4 neutropenia only in one dog in the EG after the first VBL administration. This case showed improvement in the neutrophil count after one interval week. The number of neutropenia episodes was not significantly different between groups, which suggests that $3 \mathrm{mg} / \mathrm{m}^{2}$ of VBL is safe regarding neutrophil count. A study with staggered VBL doses in dogs confirmed the safety of administering a dose of 3.5 $\mathrm{mg} / \mathrm{m}^{2}$. However, there was a higher frequency of neutropenia [1]. Another study evaluated the use of rapidly increasing VBL doses over a period of two weeks. In the aforementioned study, $70 \%$ of $34 \mathrm{dogs}$ tolerated the $3 \mathrm{mg} / \mathrm{m}^{2}$ well. Additionally, neutropenia was the most observed adverse effect and factors such as response rate and survival time were not evaluated [21].

In addition to a follow-up CBC test, the evaluation of gastrointestinal effects is important as a parameter to measure toxicity during chemotherapy MCT treatment. In the present study, gastrointestinal adverse events were mild and self-limited in both groups. Similar findings were also described in previous studies $[4,15,21,23]$.

Tumor recurrence is often an important factor when evaluating the efficacy of treatments for MCT in dogs $[9,12]$. The exact role of the VBL dose in the relapse and survival of dogs with MCT is still in debate. A study involving 35 dogs with high-grade, metastatic, or disseminated MCTs evaluated a combined therapy with $3.5 \mathrm{mg} / \mathrm{m}^{2}$ dose of VBL and prednisone. The response rate was $65 \%$ and there was recurrence in 16 dogs, with a mean disease free time of 489 days. Survival time was significantly lower for dogs with grade III MCT, indicating that the histopathological grade is an important factor associated with survival [15]. Regarding relapse after treatment in the present study, EG dogs had significantly less tumor recurrence than the dogs in the CG. This finding suggests that using larger VBL doses for the treatment of MCT in dogs might be beneficial.

The relationship between dose intensity and efficacy in veterinary oncology remains unclear for several types of tumors. A study involving dogs with lymphoma showed that higher doses of chemotherapy might promote positive therapeutic and prognostic response, although they can cause more adverse effects than the doses used in traditional protocols. The increase in dosage, however, should be gradual and often personalized [18]. One preliminary study evaluated the use of VBL for treatment of naïve multicentric lymphoma in dogs. In that study, dogs were treated with $2 \mathrm{mg} / \mathrm{m}^{2}$ doses of VBL while a small subset of dogs were treated with $2.5 \mathrm{mg} / \mathrm{m}^{2}$ doses of VBL. Dogs treated with a higher VBL dose had significantly improved response rates, suggesting that an increase in the VBL dose may improve efficacy in some cases [4].

A long follow-up period is required to establish the actual beneficial effect of a higher dose 
of VBL for the treatment of MCT [5]. Although previous studies evaluated higher doses of VBL for MCT treatment in dogs, their focus was on evaluating the toxicity and identifying safe dosages and the long-term effect of a higher dose was not an endpoint $[14,21,23]$. In the present study, the overall survival following diagnosis revealed no significant difference between EG and CG. A cross-sectional analysis of the overall survival of the dogs showed that over the period of 365 days (one year) after the histopathological diagnosis, there was also no significant difference between groups. These results suggest that higher doses of VBL do not negatively affect survival.

The importance of MCT in dogs in the field of veterinary oncology is evident in this and other studies. The variable clinical presentation consists of factors that make it difficult to choose an optimal treatment protocol. This study had limitations, which include a limited follow-up period, small sample size and absence of surgical margins evaluation data. Despite these limitations, we presented an epidemiological profile of dogs with grade II and III MCT at two different treatment centers.

\section{CONCLUSIONS}

The study evaluated the treatment of MCT over a period in dogs using a higher dose of VBL compared with controls, which received the standard dose of VBL. The comparison between groups revealed similar adverse effects. The reductions in hematological parameters did not exceed the lower limit for the species. The experimental group had a lower rate of tumor recurrence than the control group. The results suggest that higher doses of vinblastine may be possible for dogs with cutaneous MCT.

\section{MANUFACTURERS}

${ }^{1}$ Libbs Farmacêutica Ltda. São Paulo, SP, Brazil.

${ }^{2}$ Mantecorp Indústria Química e Farmacêutica Ltda. Rio de Janeiro, RJ, Brazil.

Acknowledgements. This study was financed in part by the Coordenação de Aperfeiçoamento de Pessoal de Nível Superior - Brasil (CAPES) - Finance Code 001.

Ethical approval. All the procedures employed in this study were approved by the PUCPR Ethics Committee for Animal Use under protocol 01106/2016.

Declaration of interest. The authors report no conflicts of interests. The authors alone are responsible for the content and writing of paper.

\section{REFERENCES}

1 Bailey D.B., Rassnick K.M., Kristal O., Chretin J.D. \& Balkman C.E. 2008. Phase I dose escalation of single-agent vinblastine in dogs. Journal of Veterinary Internal Medicine. 22(6): 1397-1402.

2 Blackwood L., Murphy S., Buracco P., DeVos J.P., De Fornel-Thibaud P., Hirschberger J., Kessler M., Pastor J., Ponce F., Savary-Bataille K. \& Argyle D.J. 2012. European consensus document on mast cell tumours in dogs and cats. Veterinary and Comparative Oncology. 10(3): e1-e29.

3 Davies D.R., Wyatt K.M., Jardine J.E., Robertson I.D. \& Irwin P.J. 2004. Vinblastine and prednisolone adjunctive therapy for canine cutaneous mast cell tumors. Journal of the American Animal Hospital Association. 40(2): 124-130.

4 Harding K., Bergman N., Smith A., Lindley S., Szivek A., Milner R., Brawner W. \& Lejeune A. 2018. Response rate to single dose of vinblastine administered to dogs with treatment-naive multicentric lymphoma. Veterinary and Comparative Oncology. 16(4): 636-641.

5 Hayes A., Adams V., Smith K., Maglennon G. \& Murphy S. 2007. Vinblastine and prednisone chemotherapy for surgically excised grade III canine cutaneous mast cell tumors. Veterinary and Comparative Oncology. 5(3): 168-176.

6 Horta R.S., Lavalle G.E., Monteiro L.N., Souza M.C., Cassali G.D. \& Araujo R.B. 2018. Assessment of canine mast cell tumor mortality risk based on clinical, histologic, immunohistochemical, and molecular features. Veterinary Pathology. 55(2): 212-223.

7 Kiupel M., Webster J.D., Bailey K.L., Best S., DeLay J., Detrisac C.J., Fitzgerald S.D., Gamble D., Ginn P.E., Goldschmidt M.H., Hendrick M.J., Howerth E.W., Janovitz E.B., Langohr I., Lenz S.D., Lipscomb T.P., Miller M.A., Misdrop W., Moroff S., Mullaney T.P., Neyens I., O`Toole D., Ramos-Vara J., Scase T.J., Schulman F.Y., Sledge D., Smedley R.C., Smith K., Snyder P.W., Southorn E., Stedman N.L., Steficek B.A., Stromberg P.C., Valli V.E., Weisbrode S.E., Yager J., Heller J. \& Miller R. 2011. Proposal of a 2-tier histologic grading system for canine cutaneous mast cell tumors to more accurately predict biological behavior. Veterinary Pathology. 48(1): 147-155.

8 Miller A.G., Morley O.S., Rao S., Avery A.C., Lana S.E. \& Olver C.S. 2009. Anemia is associated with decreased survival time in dogs with lymphoma. Journal of Veterinary Internal Medicine. 23(1): 116-122. 
9 Miller R.L., Lelyveld S.V., Warland J., Dobson J.M. \& Foale R.D. 2014. A retrospective review of treatment and response of high risk mast cell tumours in dogs. Veterinary and Comparative Oncology. 14(4): 361-370.

10 Mochizuki H., Motsinger-Reif A., Bettini C., Moroff S. \& Breen M. 2016. Association of breed and histopathological grade in canine mast cell tumours. Veterinary and Comparative Oncology. 15(3): 829-839.

11 Mullins M.N., Dernell W.S., Withrow S.J., Ehrhart E.J., Thamm D.H. \& Lana S.E. 2006. Evaluation of prognostic factors associated with outcome in dogs with multiple cutaneous mast cell tumors treated with surgery with and without adjuvant treatment: 54 cases (1998-2004). Journal of the American Animal Hospital Association. 228(1): 91-95.

12 Olsen J.A., Thomson M., O`Connell K. \& Wyatt K. 2018. Combination vinblastine, prednisolone and toceranib phosphate for treatment of grade II and III mast cell tumours in dogs. Veterinary Medicine and Science. 4(3): 237-251.

13 Patnaik A.K., Ehler W.J. \& MacEwen E.G. 1984. Canine cutaneous mast cell tumor: morphologic grading and survival time in 83 dogs. Veterinary Pathology. 21(5): 469-474.

14 Rassnick K.M., Bailey D.B., Flory A.B., Balkman C.E., Kiselow M.A., Intile J.L. \& Autio K. 2008. Efficacy of vinblastine for treatment of canine mast cell tumors. Journal of Veterinary Internal Medicine. 22(6): 1390-1396.

15 Rassnick K.M., Bailey D.B., Russell D.S., Flory A.B., Kiselow M.A., Intile J.L., Malone E.K., Balkman C.E. \& Barnard S.M. 2010. A phase II study to evaluate the toxicity and efficacy of alternating CCNU and high-dose vinblastine and prednisone (CVP) for treatment of dogs with high-grade, metastatic or nonresectable mast cell tumours. Veterinary and Comparative Oncology. 8(2): 138-152.

16 Shoop S.J., Marlow S., Church D.B., English K., McGreevy P.D., Stell A.J., Thomson P.C., O`Neill D.G. \& Brodbelt D.C. 2015. Prevalence and risk factors for mast cell tumours in dogs in England. Canine Genetics and Epidemiology. 2: $1-10$.

17 Sledge D.G., Webster J. \& Kiupel M. 2016. Canine cutaneous mast cell tumors: A combined clinical and pathologic approach to diagnosis, prognosis, and treatment selection. The Veterinary Journal. 215: 43-54.

18 Sorenmo K., Overley B., Krick E., Ferrara T., LaBlanc A. \& Shofer F. 2010. Outcome and toxicity associated with a dose-intensified, maintenance-free CHOP-based chemotherapy protocol in canine lymphoma: 130 cases. Veterinary and Comparative Oncology. 8(3): 196-208.

19 Stefanello D., Buracco P., Sabattini S., Finotello R., Giudice C., Grieco V., Iussich S., Tursi M., Scase T., DiPalma S., Bettini G., Ferrari R., Martano M., Gattino F., Marrington M., Mazzola M., Elisabetta Vasconi M., Annoni M. \& Marconato L. 2015. Comparison of 2- and 3-category histologic grading systems for predicting the presence of metastasis at the time of initial evaluation in dogs with cutaneous mast cell tumors: 386 cases (2009-2014). Journal of the American Animal Hospital Association. 246(7): 765-769.

20 Thamm D.H., Turek M.M. \& Vail D.M. 2006. Outcome and prognostic factors following adjuvant prednisone/vinblastine chemotherapy for high-risk canine mast cell tumour: 61 cases. Journal of Veterinary Medical Science. 68(6): 581-587.

21 Varela J.C.S., Pecceu E., Handel I. \& Lawrence J. 2016. Tolerability of a rapid-escalation vinblastine-prednisolone protocol in dogs with mast cell tumours. Veterinary Medicine and Science. 2(4): 266-280.

22 Veterinary Cooperative Oncology Group. 2016. Common Terminology Criteria for Adverse Events (VCOG-CTCAE) following chemotherapy or biological antineoplastic therapy in dogs and cats v1.1. Veterinary and Comparative Oncology. 14(4): 417-446.

23 Vickery K.R., Wilson H., Vail D.M. \& Thamm D.H. 2008. Dose-escalating vinblastine for the treatment of canine mast cell tumour. Veterinary and Comparative Oncology. 6(2): 111-119.

24 Webster J.D., Yuzbasiyan-Gurkan V., Thamm D.H., Hamilton E. \& Kiupel M. 2008. Evaluation of prognostic markers for canine mast cell tumors treated with vinblastine and prednisone. BMC Veterinary Research. 4: 32.

25 Wilson L., Jordan M.A., Morse A. \& Margolis R.L. 1982. Interaction of vinblastine with steady-state microtubules in vitro. Journal of Molecular Biology. 159(1): 125-149. 